\title{
REVISIÓN DE POLÍTICAS PÚBLICAS QUE PERMITAN POTENCIALIZAR EL DESARROLLO DE LA REGIÓN DE RÁQUIRA (COLOMBIA) EN MATERIA DE TURISMO, RECUPERANDO SUS SABERES ANCESTRALES CON LA VINCULACIÓN DE LOS DESMOVILIZADOS EN EL MARCO DEL POSCONFLICTO
}

Review of Public Policies that allow potentialize the Development of Raquira Region in Colombia, in the Field of Tourism, recovering its Ancestral Knowledge with the Incorporation of the Demobilized People in the Framework of the Post-Conflict

Ana Libia SERRANo Jaimes, JuAn de Jesús SÁNCHEZ TORRES

Universidad Militar Nueva Granada, Colombia

\section{KEY WORDS}

\section{CONPES}

Demobilized

Post-Conflict

Post-Agreement

Reinsertion

Reintegration

Ancestral Knowledge

\section{ABSTRACT}

In the post-conflict context in Colombia, new development strategies for social and labor inclusion have been defined in the marginalized regions as a result of the internal conflict that lasted more than 50 years. Today, for the whole country and specifically for the demobilized population, an opportunity to reintegrate into civil life has been opened and to be linked to the development of the regions, taking advantage of the natural resources, people's creativity and in particular the ancestral knowledge, as a factor of competitiveness and regional sustainability within the framework of the Productive Transformation Policy of the Country.

\section{RESUMEN}

\section{CONPES}

Desmovilizados

Postconflicto

Postacuerdo

Reinserción

Reintegración

Saberes ancestrales
El escenario del posconflicto en Colombia ha permitido definir nuevas estrategias de desarrollo e inclusión social y laboral para las regiones marginadas, que durante más de 50 años de confrontación interna sufrieron el abandono del estado. Hoy Colombia enfrenta nuevos retos no solo para el sector público, también para el sector privado, que visto desde una perspectiva gerencial se convierte en una oportunidad para hacer de dicha población uno de los factores que impulsen el desarrollo de las regiones al reinsertase a la vida civil. 
$\mathrm{M}$ ientras algunas regiones de Colombia quedaron deshabitadas por el desplazamiento forzado y la falta de recursos, otras regiones por su cercanía a las grandes ciudades sufrieron la misma suerte pero las causas que motivaron el éxodo de sus habitantes obedece a la falta de oportunidades laborales, la precaria educación, el aparente agotamiento de los recursos naturales y la obsolescencia de sus productos autóctonos que constituían desde siempre la base de la economía, lo que generó una escases de mano de obra y el cierre de las empresas familiares, un ejemplo de ellos es el municipio de Ráquira, en el departamento de Boyacá.

El común denominador de estas regiones se centra en la falta de mano de obra que impulse la economía y la ausencia de población que trasmita la cultura y saberes ancestrales.

En el marco de la Política de Trasformación Productiva del País- PTP, la región de Ráquira, en el departamento de Boyacá, Colombia, reúne las condiciones para desarrollar estrategias que permitan dar cabida a la población desmovilizada, aprovechando las políticas públicas en materia de competitividad y el desarrollo de las alianzas público privadas para la inclusión, su vinculación a las actividades económicas de la región impulsan el desarrollo, se convierten en garantes de la recuperación y conservación de los saberes ancestrales la cultura autóctona y a la vez contribuyen con el cumplimiento de los objetivos estratégicos del país en materia de desarrollo sostenible propuestos para el 2032, en concordancia con las metas de desarrollo de la agenda de la UNESCO para el 2030.

El presente trabajo hace parte de la investigación: "Identificación de factores competitivos claves para potenciar como destino turístico de naturaleza las áreas libres de sospecha de minas antipersona del oriente antioqueño en el posconflicto y otras regiones de Colombia", adelantado por el grupo de semillero MULTIVERSO y en alianza con facultades de otras reconocidas universidades del país.

La investigación consta de tres fases, de la cual esta es la primera, que consiste en una exploración en materia de políticas públicas en cuanto a los programas de reinserción específicamente en la región de Ráquira, Boyacá, región que ofrece oportunidades de mano de obra y recursos naturales para desarrollar programas de iniciativa comunitaria.

La segunda fase es de tipo exploratoria de campo, para caracterizar la región en su capacidad de oferta laboral e identificar los saberes ancestrales que se están perdiendo por falta de generaciones jóvenes que los reciban.

La tercera fase es la identificación y valoración de factores competitivos que permitan desarrollar la región como destino turístico de clase mundial según la política de trasformación productiva.

La preocupación del gobierno colombiano por fomentar la competitividad y productividad para el desarrollo del país ha sido presentada desde el documento CONPES 3527 de 2008, para lograr los objetivos al año 2032:

La Comisión estableció los siguientes cinco pilares para la política de competitividad: (1) desarrollo de sectores o clústers de clase mundial (2) salto en la productividad y el empleo, (3) formalización empresarial y laboral, (4) fomento a la ciencia, la tecnología y la innovación, y (5) estrategias transversales de promoción de la competencia y la inversión (Conpes, C. N. (2008). Documento Conpes 3527, 5)

Estos cinco pilares son los que permiten generar estrategias que vinculen los sectores de la población marginada $y$ los territorios que han sido abandonados de la presencia del estado, de esta manera se logra una trasformación social globalizada enmarcada en un punto de partida que sugiere la reflexión constante desde el planteamiento y desarrollo de políticas que establezcan el direccionamiento hacia un crecimiento económico de las regiones con propósito de avance en sus territorios.

Además de proponer los cinco pilares se hace una apuesta en el citado documento desde la agenda interna para el cuatrienio de 2006-2010, como una preocupación fundamental para generar un estado comunitario, donde se desarrollen todos, sin embargo el documento aunque reconoce la importancia y la responsabilidad del sector público, no deja de lado al sector privado pero se queda corto en proponer estrategias que direccionen el papel de dicho sector para el cumplimiento de las metas:

La competitividad se entiende como un complemento a las condiciones de entorno para el crecimiento. En particular, el crecimiento tiene unas condiciones necesarias, que tienen que ver con la seguridad física y jurídica, y con la estabilidad macroeconómica. La importancia de esas condiciones es vital. En este documento no se hacen consideraciones sobre las condiciones de entorno, en el entendido de que éstas deben ser satisfechas. Una política de competitividad no sustituye a la Seguridad Democrática ni a un entorno macroeconómico sano y estable, sino que los complementa, y permite potenciar el efecto de éstos sobre el crecimiento. (Conpes, C. N. (2008). Documento Conpes 3527, 5)

En el documento 3527 se deja claro que el sector privado es el responsable de la productividad, la reflexión se centra entonces en el entorno, es aquí donde se hace necesario revisar otras políticas en materia de inclusión de la población, en especial, aquellas que por vía de la desmovilización y 
dejación de armas se quieren vincular al sector productivo; para el mismo año de 2008 el Consejo Nacional de Política Económica y Social , considera el conpes 3554, donde se plantea la política nacional de reintegración social y económica para personas y grupos armados ilegales, con la visión Colombia segundo centenario 2019, aunque existe una preocupación en materia de la vinculación de los desmovilizados al sector productivo del país no se hace ninguna referencia sobre la manera cómo el sector privado puede vincularse al la reintegración de esta población:

La Política busca asegurar la superación de su condición a través de: a) la integración de la oferta social y económica del Estado; b) el acompañamiento para incrementar la probabilidad de que las intervenciones mejoren las condiciones de calidad de vida de la población desmovilizada y de sus familias; y c) la construcción de un marco de corresponsabilidad que por un lado, apoye al desmovilizado a regresar y convivir constructivamente en su entorno familiar y comunitario, y por el otro, lo comprometa a él y a sus dependientes con la superación de su situación y la permanencia en la legalidad.( DNP, D. (2008). Consejo Nacional de Política Económica y Social-CONPES 3554: Política Nacional de Reintegración Social y Económica Para Personas y Grupos Armados Ilegales. Bogotá DC., 2)

En materia de acuerdos de paz, se aclara en el Conpes 3554 que no existe un referente a nivel mundial que se pueda comparar con el proceso de desarme, desmovilización y reintegración como el que se está haciendo en Colombia, se proponen varias estrategias para que los desmovilizados se puedan considerar reintegrados a la vida civil, se citan los decretos128 de 2003, 3043 de 2006 y 395 de 2007, donde existe la oferta del gobierno para la reinserción pero no se hace una conexión directa con el sector privado en materia de alianzas público privadas.

Las meta perseguida en el conpes 3554, es la consolidación de la paz y el desarrollo de las familias de los desmovilizados para que regresen a sus lugares de origen, pero las vías para un desarrollo competitivo como lo plantea el conpes 3527 no tienen articulación con los programas de tipo asistencial que ofrece el gobierno, quedan dichas de forma vaga y a discreción de la interpretación pero de ninguna manera comprometen a los gobiernos locales, un ejemplo es que el primer enfoque de reintegración queda de manera explícita así:

... el desarrollo de estrategias de largo plazo aplicadas a cada persona (proyecto de vida) o grupo de individuos, las cuales incluyen la promoción y desarrollo de capacidades y aptitudes académicas, vocacionales y ciudadanas necesarias para la interacción pacífica en la sociedad. Este enfoque es el más común en el mundo, dado que es menos costoso y más fácil de implementar.( DNP, D. (2008). Consejo Nacional de Política Económica y Social-CONPES 3554: Política Nacional de Reintegración Social y Económica Para Personas y Grupos Armados Ilegales. Bogotá DC., 8)

Los otros dos enfoques para la reintegración nombran solamente la importancia de crear ciudadanía y espacios de diálogo para generar nuevamente una conciencia vinculante con el nuevo entorno al que se enfrentan los desmovilizados que desean integrarse a la vida civil, además es importante resaltar que para la política nacional de reincorporación a pesar de fundamentar todo el proceso de acompañamiento a la población desmovilizada y en fase de reintegración en principios como: apropiación nacional, reconciliación, equidad y otros más, presenta barreras cuando caracteriza la población objetivo que además de la situación jurídica, está la poca experiencia en oficios formales, mal manejo del tiempo, deficiencia en el manejo del dinero, poca responsabilidad en el manejo de horarios de trabajo, analfabetismo etc.., esto según el estudio requiere un trabajo en el largo plazo, pero siempre asociado a los lugares de origen de los habitantes.

Según las estadísticas mostradas por el ministerio de defensa de 2.638 desmovilizados en el 2009 se han registrado para 2017 un número de 635, pero no se tienen evidencias de cuántos de los desmovilizados se reintegran a la vida civil y cuantos deciden volver a la vida ilegal o se integran a bandas delincuenciales, según un estudio publicado por INDEPAZ en diciembre de 2018, se han generado grupos armados focalizados que son producto de disidencias de las FARC y en algunos casos se habla que nunca existió un verdadero desarme, esto agudiza la situación ya que no se puede determinar cuál es el numero de desmovilizados que tiene voluntad de reinserción

De otra parte y siguiendo con la visión de Colombia 2032 en materia de competitividad existen en Colombia departamentos como se dijo anteriormente, que pueden contribuir al desarrollo del país pero no cuentan con la población que permita potencializar el desarrollo de la región, un ejemplo es el departamento de Boyacá que cuenta con 123 municipios, es considerado uno de los atractivos turísticos más importantes del país, es allí donde se consolida la independencia del dominio español, además tiene una diversidad de paisajes y pisos térmicos que brindan variedad de climas y cultivos para una gastronomía insuperable, el departamento se halla dividido en 13 provincias que agrupan 123 municipios, la provincia de Ricaurte cuenta con 13 municipios entre los que se cuenta Ráquira, uno de los pueblos con mayor atractivo turístico, su nombre se remonta a la 
tradición indígena conocidos como pueblo de "olleros", ya que allí se fabricaban las ollas donde se cocinaban los alimentos, hoy cuenta con una extensión de 233 kilómetros cuadrados y una población de 12.900 habitantes, para una densidad por kilometro cuadrado de 52 habitantes, según el informe del departamento, Boyacá en cifras (2018), para el mismo informe la distribución del PIB departamental por municipio y el peso relativo municipal en el PIB departamental, así como el grado de importancia económica. Revela, por ejemplo, en el año 2015, para las principales ciudades, por cada 100 pesos generados en producción interna bruta en Boyacá, 0,6 corresponde a producción de la ciudad de Ráquira, lo que le da un grado importancia económica de 6, siendo 1 el de mayor importancia y 7 el de menor importancia, en cuanto a la tasa de desempleo del departamento aumentó del 6,2\% del año 2015 al 7,0\% para el 2016, según datos suministrados por el DANE la Dirección de Síntesis y Cuentas Nacionales. Consulta: febrero de 2018.

En cuanto a la problemática por desplazamiento y desmovilización de grupos armados, según el plan de desarrollo municipal de Ráquira 2016-2019, no se han presentado ingresos masivos de población desplazada y la alcaldía le apuesta a que existe una buena disposición para contribuir a la reinserción de los desmovilizados, una gran dificultad que atraviesa el municipio es el grado de contaminación por el desarrollo de la alfarería, actualmente es el municipio más contaminado del país, según el informe presentado por el ministerio del medio ambiente a través del IDEAM en febrero del 2018, el uso de carbón mineral y madera para cocinar los productos de barro ha generado una carga de contaminación en el medio ambiente que está afectando la salud pública, las autoridades encargadas como la CAR está trabajando en estrategias para mitigar el impacto ambiental por las practicas de los artesanos de más de 350 artesanos que viven de la elaboración de productos de barro, a esto se suma que los artesanos del barro son personas que aun laboran con las técnicas ancestrales heredadas de sus abuelos pero ya las nuevas generaciones de la región no están interesadas en seguir el oficio, lo que ha llevado a que se pierdan técnicas milenarias de fabricación de artículos de barro y consigo también la cultura trasmitida por este tipo de oficio.

Dentro del plan de desarrollo municipal 20162019 en ejecución por el señor alcalde Néstor Alonso Castillo Buitrago, se identifican los retos en los que el municipio debe tomar cursos de acción prioritarios, donde el trabajo informal y el trabajo infantil, surgen a partir de la inadecuada estructuración empresarial puesto que hace necesario la reorganización de la actividad turística en la región ya que su principal actividad económica depende directamente de este sector, dentro de la problemática a nivel turístico se evidencia la falta de cultura para atender al turista, la poca importancia que se le da al artesano, la falta de puntos de información para los visitantes, la inexistencia de guías y mapas turísticos, y la falta de apoyo para impulsar la comercialización de la artesanía.

A raíz de la interacción que se tuvo con la comunidad se generaron aspectos positivos a tener en cuenta, la administración municipal y sus habitantes tienen el compromiso de darle un rumbo diferente a la región en pro del bienestar de su economía y el de cada persona que reside en el lugar.

En el año 2009 se crean iniciativas conjuntas para el impulso y la promoción del patrimonio artesanal y el turismo colombiano (política de turismo de artesanías ( 2009), en conjunto con Artesanías de Colombia SA se crea un valor agregado a la artesanía hecha en las diferentes regiones del país, lo que infiere en ser un elemento importante a la hora de que el turista disfruta de la visita y quiere simbolizar su experiencia a través de un elemento material. Además de buscar la contribución al progreso del sector artesanal esta política dentro de su gestión rigen varias estrategias a implementar como lo son: la identificación y la valoración de las ofertas artesanales de los principales destinos turísticos, coordinar acciones con los prestadores de servicios turísticos para incluir las ofertas artesanales dentro de los planes turísticos, incentivar en la educación media el aprendizaje de los saberes, expresiones y técnicas artesanales, introducir parámetros de sostenibilidad dentro del trabajo artesanal en este caso con cerámica.

Frente a la comercialización esta política tiene el propósito de establecer estándares de calidad en la producción de producto artesanal así mismo la responsabilidad social y sostenibilidad para los prestadores de servicios turísticos, generar espacios innovadores para propiciar nuestras artesanales generando la comercialización de los productos. También se deben buscar alianzas estratégicas para buscar apoyo con los entes encargados de la gestión turística. (Política de Artesanías, 2009).

Otra de las estrategias fundamentales para garantizar el desarrollo y la implementación de políticas que respaldan el turismo es la seguridad que se debe adoptar en el sector, teniendo en cuenta un conjunto de medidas de control hacia la prevención y la mitigación de los riesgos que se puedan presentar tanto para el visitante como para las diferentes entidades y personas prestadoras del servicio. Para ello se realiza la revisión del plan estratégico de seguridad turística. En conjunto con la policía de turismo de Colombia donde su objetivo principal es incrementar la seguridad de los turistas a partir de proyectos y actividades que promuevan medidas de prevención, vigilancia de los atractivos 
turísticos y orientación al turista, a su vez logrando un fortalecimiento institucional (Plan Estratégico de Seguridad Turística, 2016) .

Colombia está en un proceso donde el objetivo es devolverle al país la confiabilidad en materia de seguridad, al correlacionar el presente proceso de paz en curso, el país se abre a las nuevas oportunidades de inversión. Posibilita el aumento de visitantes y de la misma forma genera innovación en lo que puede llegar a ser una gran fortaleza competitiva del país.

El plan estratégico de seguridad turística define ocho estrategias a desarrollar donde se inicia con el control y prevención de los servicios turísticos el cual se basa en generar una cultura de seguridad en el mercado turístico nacional, llevando a cabo la normatividad vigente y estandarizado los derechos y los deberes establecidos para el sector, tomando cursos de acción en la prestación de un servicio responsable. Como segunda medida se debe vigilar los atractivos y actividades turísticas, llevando a cabo la protección adecuada respecto al servicio y a la satisfacción del visitante. También se debe fortalecer la manera de informar y orientar al turista para evitar situaciones que lo pongan en peligro como la estafa o ser engañados por algún otro medio. Frente a esta estrategia es de vital importancia establecer un punto de información al turista en el municipio de Ráquira porque en el departamento de Boyacá aún no se cuenta con ello, según la ruta nacional solo existen cuatro puntos y están ubicados en sus afluentes turísticos más relevantes. La capacitación en temas como servicio al cliente, ecología, idiomas y normatividad hasta el momento no es suficiente por parte de la policía turística ni en en el municipio de Ráquira por ello es otra estrategia a evaluar para la debida ejecución. Y contar con una avanzada seguridad en las vías y rutas turísticas marca una diferencia en lo que puede experimentar el turista durante su visita.

Por otra parte, uno de los lineamientos en materia de competitividad lo afianza el programa de transformación productiva PTP, este tiene por objeto ejecutar la política industrial del país a través de una gestión entre las empresas públicas y privadas afín de eliminar las restricciones en el ámbito regional nacional e internacional que han detenido el avance productivo del país. Dentro del su campo de acción se tiene la implementación de nuevas metodologías internacionales, programas de capacitación en alianza con empresas educativas que permiten la orientación relevante para la certificación necesaria en el salto a mejorar los indicadores de producción. Actualmente el PTP está enfocado en 18 sectores potencialmente productivos del país (PTP, 2008). Una de sus apuestas es el sector turismo en un trabajo conjunto con el CONPES 3527, el turismo de naturaleza definido como "todo tipo de turismo basado en la naturaleza en la que la principal motivación es la observación y apreciación de la naturaleza, así como las culturas tradicionales" (OMT,2002)

Figura 1. Análisis comparativo entre seis países

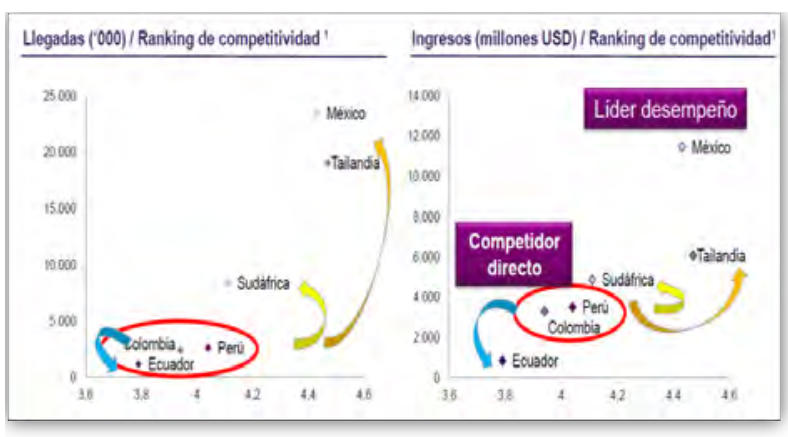

Fuente: adaptada de PTP, 2008.

En la figura 1 se observa la situación rentabilidad vs competitividad a nivel global a la cual se enfrenta Colombia, siendo el líder de desempeño México y su competidor directo Perú, con un ranking de visitantes igualitario y donde la ponderación de sus ventajas competitivas se debe ver reflejada.

Dentro de la visión de plan de negocio de turismo de naturaleza Colombia 2027 se considera como subproducto los saberes ancestrales, y se tienen líneas de acción proyectadas al fortalecimiento la promoción e innovación sectorial, incentivos para el desarrollo de infraestructura de acuerdo al marco normativo y desde el capital humano la profesionalización de las pymes alcanzando una sostenibilidad trasversal. En cuanto a la cultura del municipio de Raquira en busca de la recuperación de sus saberes ancestrales por medio de sus productos hechos en cerámica, se tiene un buen pronóstico frente a la implementación de los recursos económicos que oferta el país a través de este programa. Por otra parte en el contexto que se formuló la problemática del municipio y partiendo del trabajo infantil relacionado con la falta de mano de obra en el municipio. Se abre la posibilidad de obtener mano de obra calificada a través de otro de los retos que hoy por hoy enfrenta el país y es el proceso de inserción laboral de las personas que han dejado las armas en busca de un mejor futuro. Dentro del llamado proceso del postconflicto. 
Figura2. Histórico de personas desmovilizadas en Colombia

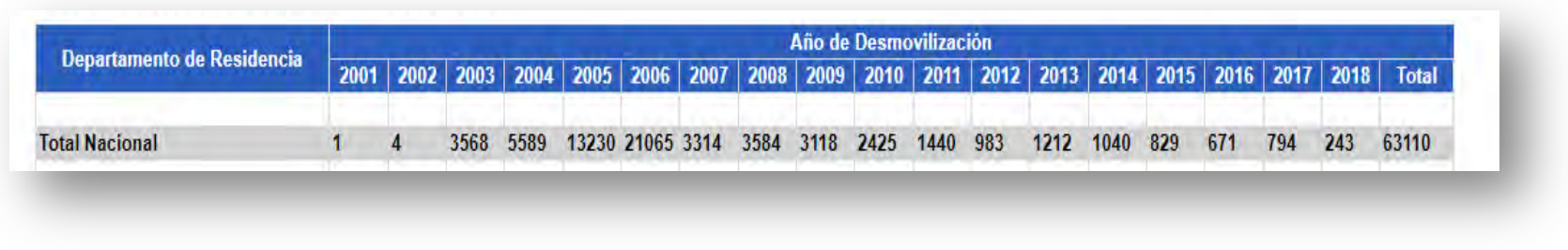

Fuente: Adaptado de Agencia Para La Reincorporación y La Normalización, 2018

En la figura 2 se observa un total de $63110^{*}$ personas desmovilizadas de grupos al margen de la ley, desde el año 2001 al año 2018, última actualización, 30 de septiembre de 2018.

A pesar de que Colombia maneja programas de reinserción y vinculación laboral de las personas que hasta el momento han dejado las armas. No se tiene por cubierto en su totalidad la reinserción frente al postconflicto por falta de oportunidades del sector empresarial.

Figura 3. Situación frente al proceso de reintegración

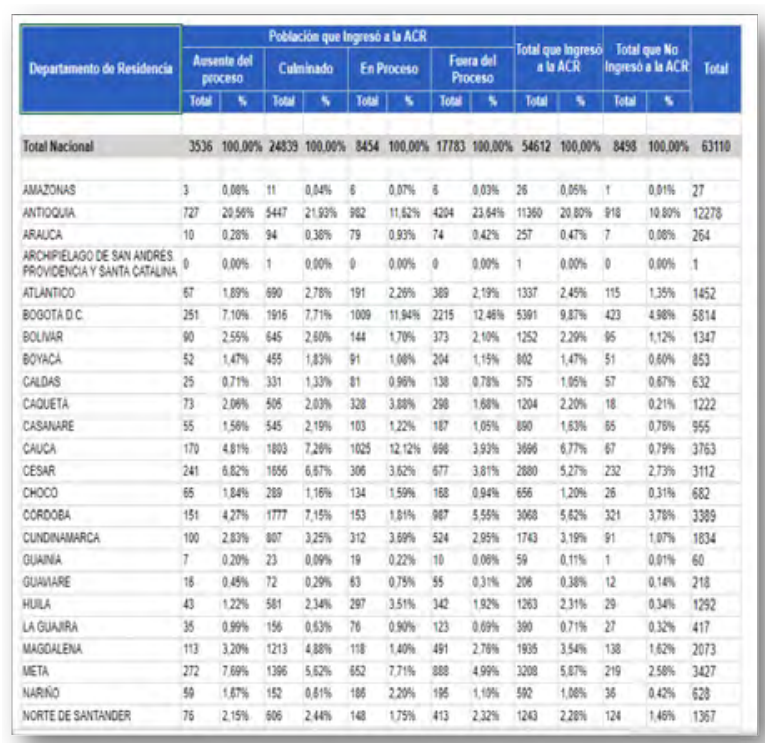

Fuente: Adaptado de Agencia Para La Reincorporación y La Normalización, 2018.
En la figura 3 se muestra la situación actual frente al proceso de reintegración de los desmovilizados del postconflicto desde la ARN donde se muestra que en el departamento de Boyacá existen 52 personas ausentes del proceso y 204 personas fuera del mismo. Frente al panorama nacional un gran número de personas aún no han tenido la oportunidad de reintegrarse a la vida laboral sin embargo cabe resaltar el esfuerzo realizado por el sector empresarial colombiano para su culminación. Desde lo descrito anteriormente se podría definir una población interesada en vincularse laboralmente a través del proceso de recuperación de los saberes ancestrales del municipio de Ráquira.

Con lo anteriormente descrito existe la posibilidad de generar estrategias que vinculen los intereses planteados desde los documentos de política pública conpes, 3554 y 3527 de 2008, para la consolidación de la paz en el país y la recuperación de los saberes ancestrales en la región de Ráquira, dando oportunidades reales a la población de desmovilizados y que desean reincorporarse a la vida civil, se superan de esta manera algunas barreras que se habían planteado en el proceso de reintegración. 


\section{Referencias}

Álvarez, Á., Ginneth, I., Alarcón, D., Duván, S., \& Sepúlveda Pinto, P. M. (2018). Análisis de los programas desarrollados por la Agencia para la Reincorporación y Normalización en la dimensión de producto (Doctoral dissertation, Corporación Universitaria Minuto de Dios).

Agencia colombiana para la Reintegración de Personas y Grupos Alzados en Armas. 31 de mayo de 2011. Resolución 163 de 2011. Recuperado el 7 de julio de 2012, de Agencia Colombiana para la Reintegración: Documents/pdf_normatividad/decretos/RESOLUCION_163_DE_2011.pdf

Agencia colombiana para la reintegración de personas y grupos alzados en armas (ACR). (2014). Caracterización de usuarios - personas en proceso de reintegración. Bogotá D.C: Sistema integrado de Gestión para la Reintegración. Recuperado a partir de http://www.reintegracion.gov.co/es/la-reintegracion/centro-dedocumentacion/Documentos/Caracterizaci\%C3\%B3n\%20Usuario\%20\%20personas\%20en\%20Proceso\%20de\%20Reintegraci\%C3\%B3n.pdf

Castillo Ramírez, A. J., \& Moreno Parga, A. (2013). La reintegración económica de personas desmovilizadas de grupos armados ilegales, como estrategia de superación de vulnerabilidades y desarrollo de capacidades.

Colombia, L. 1450 de 2011, por la cual se expide el Plan Nacional de Desarrollo 2010-2014, 48102 Diario Oficial, 16 de junio de 2011.

CONPES 3554. (2008). Política nacional de reintegración social y económica para personas y grupos armados ilegales [National Policy of Social and Economic Reintegration of Individuals and Illegal Armed Groups].

Para la Competitividad, A. C. P. (2008). La Productividad, Ministerio de Comercio, Industria y Turismo, Consejo Privado de Competitividad y Departamento Nacional de Planeación, Documento CONPES 3527. Política Nacional de Competitividad y Productividad.

De Cultura, L. Ley 397 de 1997. (1997). Recuperada de http://www. Sinic. Gov. Co/SINIC/Sipa Conceptos_Comite_Tecnico/ley, 20397, 20.

De Tunja, C. D. C., \& Duitama, S. (2007). Boyacá en Cifras. Tunja: Planeación.

De Ráquira, A. (2019). Plan de Desarrollo Municipal de Ráquira 2013-2019. Boyacá, Colombia.

Enríquez, N. (2011, mayo 4). «No habrá reconocimiento político para terroristas»: Min defensa. Recuperado a partir de http://www.eltiempo.com/archivo/documento/CMS-9275121

Escallón Romero, J. C. Centro de producción y capacitación artesanal en Ráquira (Bachelor's thesis, Facultad de Arquitectura y Diseño

El congreso de Colombia. Disposiciones para facilitar el diálogo y la suscripción de acuerdos con grupos armados organizados al margen de la ley para su desmovilización, reconciliación entre los colombianos y la convivencia pacífica., Pub. L. No. Ley 782 de 2002 (2002). Recuperado a partir de http://www.alcaldiabogota.gov.co/sisjur/normas/Norma1.jsp?i=6677

El congreso de Colombia. Por medio de la cual se prorroga la vigencia de la Ley 418 de 1997 prorrogada y modificada por las Leyes 548 de 1999 y 782 de 2002 y se modifican algunas de sus disposiciones., Pub. L. No. Ley 1106 de 2006 (2006). Recuperado a partir de http://www.secretariasenado.gov.co/senado/basedoc/ley_1106_2006.html

Mejía, L. (2014). La reintegración social y económica de los grupos armados ilegales en Colombia: reflexiones a partir de la trayectoria de nueve excombatientes (Maestría en Estudios Políticos e Internacionales). Universidad colegio mayor de nuestra señora del rosario, Bogotá D.C.

Molina Torres, P. A., \& Huertas Rodríguez, J. P. (2017). Estudio de Prefactibilidad para la Posible Implementación de Atrapanieblas en el Municipio de Ráquira.

Para la Competitividad, A. C. P. (2008). La Productividad, Ministerio de Comercio, Industria y Turismo, Consejo Privado de Competitividad y Departamento Nacional de Planeación, Documento CONPES 3527. Política Nacional de Competitividad y Productividad.

Ministerio de Comercio, Industria y Turismo/Viceministro de Ministerio de Comercio,

Industria y Turismo Gobierno de Colombia/Competitividad El desafío para alcanzar un turismo dé Clase Mundial/Política de Turismo de Artesanías /pdf/ (2009) /Política de Turismo Cultural (2007)/Política de Turismo de Naturaleza (2012)/Plan Estratégico de Seguridad Turística (2016). Recuperado de http://www.mincit.gov.co

http://raquiraboyaca.micolombiadigital.gov.co/sites/raquiraboyaca/content/files/000061/3024_pdmraquirab oy20162019webok.pdf

http://ccomerciotunja.org.co/new/wp-content/uploads/2018/06/BOYACA-EN-CIFRAS_2018-2.pdf

https://www.eltiempo.com/archivo/documento/CMS-3995750

http://www2.unwto.org/es

http://www.mincit.gov.co/minturismo/calidad-y-desarrollo-sostenible

http://www.raquira-boyaca.gov.co 